\title{
Pion family in AdS/QCD: The next generation from configurational entropy
}

\author{
Luiz F. Ferreira ${ }^{1,2, *}$ and R. da Rocha ${ }^{1, \dagger}$ \\ ${ }^{1}$ Federal University of ABC, Center of Mathematics, Santo André 09210-580, Brazil \\ ${ }^{2}$ Federal University of ABC, Center of Physics, Santo André 09210-580, Brazil
}

(Received 13 February 2019; published 1 April 2019)

\begin{abstract}
Using two-flavor AdS/QCD, with chiral and gluon condensates, we describe the pion family and its mass spectra. Using gravity-dilaton-gluon backgrounds, entropic Regge-like trajectories for the pion family are then derived. They relate the underlying configurational entropy of the $\pi$ mesons to both the pion excitation wave mode number and the pion experimental mass spectra, yielding a reliable prediction for the mass spectra of higher-excitation pion modes, which could be experimentally detected.
\end{abstract}

DOI: 10.1103/PhysRevD.99.086001

\section{INTRODUCTION}

The configurational entropy (CE) apparatus consists in measuring the shape complexity of any localized system $[1,2]$. The information encoded in random processes is represented by the $\mathrm{CE}$, which thus represents the compression of information in the wave mode configurations of any system in nature $[1,2]$. The CE setup has been shown to be an advantageous instrument to probe various aspects of $\mathrm{QCD}$, in the holographic AdS/QCD correspondence, from both theoretical and phenomenological viewpoints. Elementary particles and their excitations, and some of their features, in QCD, were shown to correspond to critical points of the CE [3-9]. Among other fields, mesonic states and glueballs play a prominent role in the informational aspects of AdS/QCD. The phenomenological dominance and abundance of certain excitation levels of mesonic states -in particular light-flavor mesons and quarkonia-and glueballs is an immediate implication of encoding and compressing information into the Fourier wave modes that underlie and constitute such states. Quantum states whose $\mathrm{CE}$ is bigger were shown to be less observable in experiments. Phenomenological aspects of the CE in AdS/QCD were studied in Refs. [3,8] for light-flavor mesons, in Refs. [6,9] for bottomonium and charmonium, in both the quantum field theory and finite-temperature setups, and in Ref. [4] for scalar glueballs. Other aspects of the CE in QCD were studied in Refs. [10-14] and in the standard model as well, in Refs. [15,16]. Informational entropic

\footnotetext{
*luizfaulhaber@if.ufrj.br

†roldao.rocha@ufabc.edu.br
}

Published by the American Physical Society under the terms of the Creative Commons Attribution 4.0 International license. Further distribution of this work must maintain attribution to the author(s) and the published article's title, journal citation, and DOI. Funded by SCOAP.
Regge-like trajectories of the $a_{1}, f_{0}$ and $\rho$ light-flavor mesons were also introduced in Ref. [17], in the context of the CE. In addition, the CE is a sharp tool that can also probe phase transitions, identified as CE critical points, in diverse systems in physics [18-29]. A more precise review of information theory, in both its classical and quantum aspects, can be found in Ref. [30].

In the last years, the AdS/QCD models have been used to study many nonperturbative aspects of QCD, such as confinement [31-34], hadronic mass spectra [35,36] and chiral symmetry breaking $[37,38]$. The AdS/QCD setup, inspired by the gauge/gravity duality [39], considers a similar type of duality, where the conformal invariance is broken after introducing an energy parameter in the AdS bulk. The simplest AdS/QCD model is the hard-wall model [40-42] and consists in placing a hard geometrical cutoff in the AdS space. Another AdS/QCD model is represented by the softwall technique [31], which reproduces a very important feature: the so-called linear Regge behavior. In this case, the background involves the AdS space and a scalar field, the dilaton, that effectively acts as a smooth infrared cutoff.

Among the mesons that can be investigated in the AdS/ QCD models, the pseudoscalar $\pi$ meson, the pion, occupies a prominent position. There have been many studies of the properties of the pion using holographic approaches [35,43-49]. In particular, the approach of Ref. [35] constructs a dynamical holographic QCD model for the twoflavor theory, in the graviton-dilaton background, that incorporates the Regge behavior of the hadron spectra, the linear quark potential and the chiral symmetry breaking. It was found in this model that the lowest pion state has a mass around $140 \mathrm{MeV}$, which can be regarded as the Nambu-Goldstone boson due to the chiral symmetry breaking. The mass spectra of the pion agrees well with the experimental data [50].

Endowed with the tools of the CE and two-flavor AdS/QCD, with chiral and gluon condensate backgrounds 
coupled to gravity, the configurational entropic Regge-like trajectories for the pion family shall be derived and scrutinized. There are two types of Regge-like trajectories to be studied. The first one relates the pion family CE to the pion excitation wave mode number, whereas the second one associates the pion family $\mathrm{CE}$ to the pion experimental mass spectra. Analyzing both types of Regge-like trajectories can, then, provide a reliable prediction for the mass spectra of higher-excitation modes of $\pi$ mesonic states. This paper is organized as follows. Section II introduces the two-flavor AdS/QCD soft-wall model and the pion family equation of motion (EOM) and main features, in this context. The mass spectra of the pion family is then depicted, showing the standard Regge trajectory. Section III is devoted to studying the gluon and chiral condensate backgrounds coupled with gravity, for two dilatonic models. The $\mathrm{CE}$ is then calculated for the $\pi$ meson family, first with respect to the $n$ excitation mode number for the $\pi_{n}$ modes in the pion family. Besides, the $\mathrm{CE}$ is also computed as a function of the pion family mass spectra, generating then a second type of nonlinear Regge trajectory. Therefore the two types of configurational entropic Regge trajectories are interpolated, relating the $\mathrm{CE}$ to both $n$ and the pion mass spectra. Hence, the mass spectra of higher-excitation $\pi_{n}$ modes is inferred. It provides a reliable phenomenological prediction of the mass spectra of the next generation of higher excitations in the pion family. Section IV closes the paper with discussions, conclusions and some perspectives.

\section{TWO-FLAVOR ADS/QCD AND THE PION FAMILY}

Meson families were comprehensively described in AdS/QCD, both in the hard- and soft-wall models [31,38,46,51-54]. Dynamical holographic models were also approached in Refs. [8,55-58]. The so-called Regge trajectories, relating the excitation number of a light-flavor mesonic state and its mass, were originally derived in Ref. [31]. Aiming to focus our approach on the pion family in the AdS/QCD, the bulk $\mathrm{AdS}_{5}$ metric is an important ingredient, reading

$$
d s^{2}=g_{M N} d \mathrm{x}^{M} d \mathrm{x}^{N}=e^{2 A(\mathrm{z})}\left(\eta_{\mu \nu} d \mathrm{x}^{\mu} d \mathrm{x}^{\nu}+d \mathrm{z}^{2}\right),
$$

where the warp factor $e^{A(\mathrm{z})}=-\mathrm{z} / \ell$, and $\eta_{\mu \nu}$ represents the boundary metric, where $\ell$ is the $\operatorname{AdS}_{5}$ curvature radius. Mesonic excitations can be described by $\mathbb{X}(\mathrm{z})$ fields in the $\mathrm{AdS}_{5}$ bulk. These fields represent the dual description of the $q \bar{q}$ operator, with mass $m_{\mathbb{X}}$, that is ruled by the following action [31]:

$$
S=-\int e^{-\phi(\mathrm{z})} \sqrt{-g} \operatorname{Tr} \llbracket d^{4} \mathrm{x} d \mathrm{z},
$$

where

$$
\mathbb{L} \sim D^{M} \mathbb{X} D_{M} \mathbb{X}+m_{\mathbb{X}}^{2} \mathbb{X}^{M} \mathbb{X}_{M}+\mathbb{F}_{L}^{2}+\mathbb{F}_{R}^{2},
$$

are the $\mathbb{A}_{L}^{m}$ and $\mathbb{A}_{R}^{m}$ gauge fields that drive the $\mathrm{SU}(2)_{R} \times$ $\mathrm{SU}(2)_{L}$ chiral flavor symmetry of QCD. The Yang-Mills field strengths are given by

$$
\mathbb{F}_{R, L}^{M N}=\partial^{[M} A_{R, L}^{N]}-i\left[A_{R, L}^{M}, A_{R, L}^{N}\right]
$$

where $A_{R, L}^{M}=A_{R, L}^{M c} f_{c}$, with $\left\{f_{c}\right\}(c=1,2,3)$ are $\mathrm{SU}(2)$ generators. The covariant derivative is explicitly given by $D^{M} \mathbb{X}=\partial^{M} \mathbb{X}-i \mathbb{A}_{L}^{M} \mathbb{X}+i \mathbb{X}, \mathbb{A}_{R}^{M}$. The $\mathbb{X}(\mathrm{z})$ field is constructed upon the pseudoscalar field, P, and a scalar field, $S$, as [35]

$$
\mathbb{X}(\mathrm{z})=\exp \left(i \mathrm{P}^{c} t^{c}\right)(\xi(\mathrm{z})+\mathrm{S}),
$$

where $\xi(z)$ is a vacuum expectation value that breaks chiral symmetry [35]. To describe the vector and axial-vector mesons, the left, $\mathbb{A}_{L}$, and right, $\mathbb{A}_{R}$, gauge fields can be split into vector, $\mathrm{V}$, and axial vector, A, fields, as [35]

$$
\begin{aligned}
\mathrm{A}^{M} & =\frac{1}{2}\left(\mathbb{A}_{R}^{M}-\mathbb{A}_{L}^{M}\right), \\
V^{M} & =\frac{1}{2}\left(\mathbb{A}_{R}^{M}+\mathbb{A}_{L}^{M}\right)
\end{aligned}
$$

yielding the respective gauge field strengths,

$$
\begin{aligned}
& F_{V}^{M N}=\partial^{[M} V^{N]}+i\left[V^{N}, V^{M}\right], \\
& F_{\mathrm{A}}^{M N}=\partial^{[M} \mathrm{A}^{N]}+i\left[\mathrm{~A}^{N}, \mathrm{~A}^{M}\right] .
\end{aligned}
$$

With respect to the vector $V$ and axial vector A fields, the soft-wall Lagrangian (3) reads

$$
\mathbb{L} \sim \mathrm{D}^{M} \mathbb{X} \mathrm{D}_{M} \mathbb{X}+m_{\mathbb{X}}^{2} \mathbb{X}^{M} \mathbb{X}_{M}+2\left(F_{\mathrm{A}}^{2}+F_{V}^{2}\right),
$$

for $\mathrm{D}^{M} \mathbb{X}=\partial_{M} \mathbb{X}+i\left[\mathbb{X}, V_{M}\right]-i\left\{A_{M}, \mathbb{X}\right\}$. The EOM for the $\xi(\mathrm{z})$ field then reads

$\left[\frac{d^{2}}{d \mathrm{z}^{2}}-\left(\phi^{\prime}(\mathrm{z})-3 \mathrm{~A}^{\prime}(\mathrm{z})\right) \frac{d}{d \mathrm{z}}-m_{\mathbb{X}}^{2}(\mathrm{z}) e^{2 A(\mathrm{z})}\right] \xi(\mathrm{z})=0$.

The analytical expression, as well as the plots of the $\xi(\mathrm{z})$ field can be found in Refs. [17,35]. We denote by $\pi_{n}$ the functions that describe the pions, and by $\varphi_{n}$ those that represent the $\phi$ mesons. It is worth emphasizing that, as in Ref. [17], the $\pi_{n}(\mathrm{z})$ modes are regarded as the quantum-mechanical wave functions, $\psi_{n}(\mathrm{z})$, by $\pi_{n}(\mathrm{z})=z^{1 / 2} \exp \left(\mathrm{z}^{2} / 2\right) \psi_{n}(\mathrm{z})$. The index $n=1$ often denotes the ground state. Therefore, the functional form for $\psi_{n}$ reads $\psi_{n}(\mathrm{z}) \sim$ $e^{-\mathrm{z}^{2} / 2} \mathrm{z}^{m+1 / 2} L_{n-1}^{m}\left(\mathrm{z}^{2}\right)$, corresponding to the $n=1$ level for the standard ground state, taking the same indexing as 
the hydrogen radial quantum number problem. The EOMs driving the pion and $\phi$ meson families read

$$
\begin{aligned}
& \left(-\frac{d^{2}}{d \mathrm{z}^{2}}+V_{\pi}(\mathrm{z})\right) \pi_{n}(\mathrm{z})=m_{n}^{2}\left(\pi_{n}(\mathrm{z})-e^{A} \xi \varphi_{n}(\mathrm{z})\right), \\
& \left(-\frac{d^{2}}{d \mathrm{z}^{2}}+V_{\varphi}(\mathrm{z})\right) \varphi_{n}(\mathrm{z})=e^{A} \xi\left(\pi_{n}(\mathrm{z})-e^{A} \xi \varphi_{n}(\mathrm{z})\right),
\end{aligned}
$$

where the Schrödinger-like potentials are respectively given by

$$
\begin{aligned}
V_{\pi}(\mathrm{z})= & \frac{\mathrm{A}^{\prime \prime}}{2}-\frac{\phi^{\prime \prime}}{2}+\frac{\xi^{\prime \prime}}{\xi}-(\log \xi)^{\prime 2} \\
& +\frac{1}{4}\left(\phi^{\prime}-3 \mathrm{~A}^{\prime}-2(\log \xi)^{\prime}\right)^{2}, \\
V_{\varphi}(\mathrm{z})= & -\frac{\phi^{\prime \prime}-\mathrm{A}^{\prime \prime}}{2}+\frac{\left(\phi^{\prime}-\mathrm{A}^{\prime}\right)^{2}}{4} .
\end{aligned}
$$

Endowed with the scalar potential (13), the mass spectra of the pion family can be read off from Eq. (11). As the only element in the $\phi_{n}$ meson family that was experimentally observed is the $\phi(1020)$, whereas the $\phi(1680)$ is not yet included in the Particle Data Group (PDG) summary table, the analysis concerning the pion family shall be emphasized in what follows. In fact, experimental data regarding the $\phi_{n}$ family is very scarce. The experimental mass spectra of the pion family is depicted in Fig. 1. The pseudoscalar mesons $\pi_{0}, \pi_{ \pm}, \pi(1300)$, and $\pi(1800)$ have already been experimentally confirmed. On the other hand, the $\pi(2070)$ and $\pi(2360)$ excitations are omitted from the PDG summary

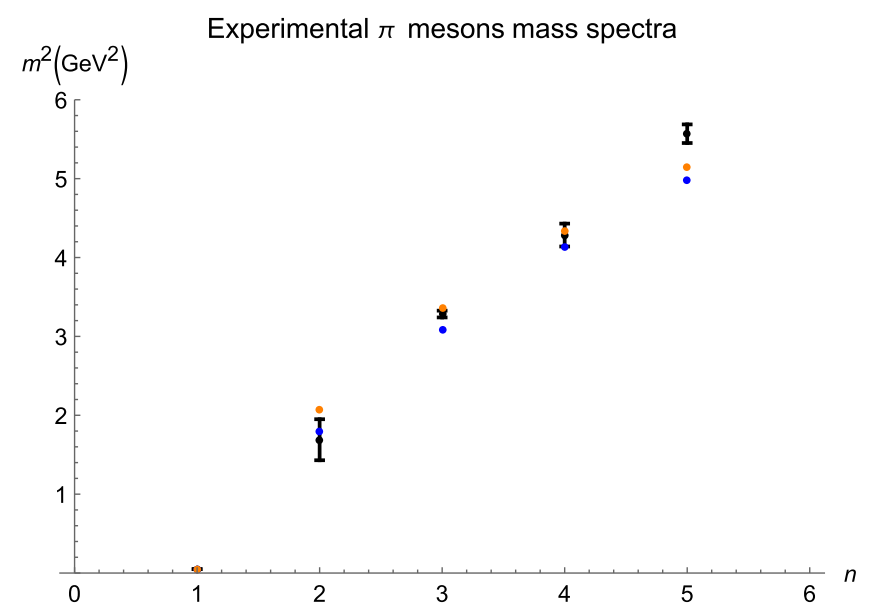

FIG. 1. Pion mass spectra as a function of the excitation number $n$ : experimental values (black points) and the AdS/QCD model predictions for the background modification given by Eqs. (15) (blue points) and (16) (orange points). The pseudoscalar mesons $\pi_{0}, \pi_{ \pm}, \pi(1300)$, and $\pi(1800)$ take their PDG values. The last two states $\pi(2070)$ and $\pi(2360)$ are omitted from the PDG summary table [50]. table [50]. The Regge trajectory for the pion meson family can be extrapolated from Fig. 1.

\section{CONFIGURATIONAL ENTROPIC REGGE TRAJECTORIES IN TWO-FLAVOR ADS/QCD}

The AdS/QCD setup can be then employed to derive configurational entropic Regge trajectories for the pion family. Informational Regge trajectories were studied [17] for the $a_{1}, f_{0}$ and $\rho$ meson families using a two-flavor softwall model, with gluon and chiral condensates, coupled to gravity with a dilaton [33]. The following dilatons were introduced in Refs. $[17,35]$ to model mesons and glueballs:

$$
\begin{aligned}
& \phi_{1}(\mathrm{z})=\mu_{\mathrm{G}}^{2} \mathrm{Z}^{2}, \\
& \phi_{2}(\mathrm{z})=\mu_{\mathrm{G}}^{2} \mathrm{z}^{2} \tanh \left(\mu_{\mathrm{G}^{2}}^{4} \mathrm{z}^{2} / \mu_{\mathrm{G}}^{2}\right) .
\end{aligned}
$$

These will be employed as the prototypical dilaton in softwall AdS/QCD [31] [Eq. (15)] and its deformation [Eq. (16)], respectively. The deformed dilaton in the UV limit yields the quadratic dilaton. The holographic gluon condensate is dual to the quadratic dilaton (15) and has an energy scale $\mu_{\mathrm{G}}$ when it corresponds to a dimension-two system, whereas it has an energy scale $\mu_{\mathrm{G}}^{2}$ when describing a dimension-four dual system [38,52]. A graviton-gluondilaton action in AdS can be given by [35],

$$
\begin{aligned}
S= & \kappa_{5}^{2} \int \sqrt{-g} e^{-2 \phi}\left\{\left[R+4 \partial^{M} \phi \partial_{M} \phi-4 V_{g}(\phi)\right.\right. \\
& \left.\left.-16 \lambda e^{-\phi}\left(\partial^{M} \xi \partial_{M} \xi+V(\phi, \xi)\right)\right]\right\} d^{5} x,
\end{aligned}
$$

where $\lambda$ denotes a general coupling, and $V_{g}$ denotes the gluon system potential. Reference [35] studied a heavy quark potential in the background given by Eq. (17), deriving the physical effective potential $V(\phi, \xi) \approx \xi^{2} \phi^{2}$. For both $\phi_{1}(\mathrm{z})$ and $\phi_{2}(\mathrm{z})$ [Eqs. (15) and (16), respectively], the parameters $\mu_{\mathrm{G}^{2}}=\mu_{\mathrm{G}} \approx 0.431$ were adopted in Refs. [17,35], in full compliance with PDG experimental data. Numerical analysis of the EOMs derived from Eq. (17), in Ref. [35], yielded the solutions for $\xi(z)$, for both the dilatonic backgrounds. The first column in Table I lists the PDG 2018 mass spectra values for $\pi_{1}=\left\{\pi_{ \pm}, \pi_{0}\right\}$, $\pi_{2}=\pi(1300)$, and $\pi_{3}=\pi(1800)$, as well as for $\pi_{4}=$ $\pi(2070)$ and $\pi_{5}=\pi(2360)$ that are still omitted from the PDG summary table (too few events measured [50]).

Table I shows the pseudoscalar pion family, identifying the $\pi_{n}$ eigenfunctions in Eq. (12), as $\pi_{1}=\left\{\pi_{ \pm}, \pi_{0}\right\}$, $\pi_{2}=\pi(1300), \pi_{3}=\pi(1800), \pi_{4}=\pi(2070), \pi_{5}=\pi(2360)$, whereas the other states have not been experimentally confirmed [50]. Besides, the pseudoscalar sector can be implemented by considering the following action, pion and $\phi$ meson wave functions: 
TABLE I. Mass spectra for the pseudoscalar pion family, in the $\phi_{2}(\mathrm{z})=\mathrm{z}^{2} \tanh \left(\mu_{\mathrm{G}^{2}}^{4} \mathrm{z}^{2} / \mu_{\mathrm{G}}^{2}\right)$ dilaton, for the mesons $\pi_{0}, \pi(1300)$, $\pi(1800), \pi(2070)$, and $\pi(2360)$. The modes indicated with an asterisk are not established particles and therefore are omitted from the PDG summary table.

\begin{tabular}{lccc}
\hline \hline & Pseudoscalar pion mass & spectra $(\mathrm{MeV})$ & \\
\cline { 2 - 4 }$n$ & Experimental & mass $_{\phi_{1}(\mathrm{z})}$ & mass $_{\phi_{2}(\mathrm{z})}$ \\
\hline 1 & $139.57018 \pm 0.00035$ & 139.3 & 139.6 \\
1 & $134.9766 \pm 0.0006$ & 139.3 & 139.6 \\
2 & $1300 \pm 100$ & 1343 & 1505 \\
3 & $1816 \pm 14$ & 1755 & 1832 \\
$4^{*}$ & 2070 & 2006 & 2059 \\
$5^{*}$ & 2360 & 2203 & 2247 \\
\hline \hline
\end{tabular}

$$
\begin{aligned}
S_{\pi}^{(2)}= & -\frac{1}{3 L^{3}} \int d^{5} x e^{-\phi} \sqrt{g}\left(\xi^{2} \partial^{\mathrm{z}} \pi \partial_{\mathrm{z}} \pi\right. \\
& \left.+\xi^{2} \partial^{\mu}(\varphi-\pi) \partial_{\mu}(\varphi-\pi)+L^{2} \partial^{\mathrm{z}} \partial^{\mu} \varphi \partial_{\mathrm{z}} \partial_{\mu} \varphi\right) .
\end{aligned}
$$

It is observed that in the graviton-dilaton-scalar system, the lowest pseudoscalar state has a mass around $140 \mathrm{MeV}$, which can be regarded as the Nambu-Goldstone boson due to the chiral symmetry breaking. The higher excitations yield a Regge trajectory, in full compliance with experimental data. It is worth mentioning that for both dilatonic field backgrounds (15)-(16) the so-called Gell-MannOakes-Renner relation, stating that the square of the pion mass is proportional to the product of a sum of quark masses and the quark condensate, is satisfied, yielding the production of massless pions in the $m_{q}=0$ limit, where $m_{q}$ denotes the quark mass $[43,44]$.

As the CE encodes how information measures the shape complexity of a physical system [18,19,27], computing the $\mathrm{CE}$ underlying the pion family requires its energy density, $\epsilon(\mathrm{z})$. This can be derived from a Lagrangian density, $\mathbb{L}$, with stress-momentum tensor components given by

$$
T_{M N}=\frac{2}{\sqrt{-g}}\left[\frac{\partial(\sqrt{-g} \llbracket)}{\partial g^{M N}}-\partial_{\mathrm{x} Q} \frac{\partial(\sqrt{-g} \llbracket)}{\partial\left(\frac{\partial g^{M N}}{\partial \mathrm{x}^{R}}\right)}\right] .
$$

Hence, the energy density is read off from the $T_{00}(\mathrm{z})$ tensor component in Eq. (19). The Fourier transform $\epsilon(k)=\int_{\mathbb{R}} \epsilon(\mathrm{z}) e^{-i k \mathrm{z}} d \mathrm{z}$, with respect to the $\mathrm{z}=1 / \Lambda_{\mathrm{QCD}}$ dimension that defines the $\Lambda_{\mathrm{QCD}}$ energy scale, defines the so-called modal fraction $[2,19]$

$$
\epsilon .(k)=\frac{|\epsilon(k)|^{2}}{\int_{\mathbb{R}}|\epsilon(k)|^{2} d k} .
$$

It consists of a probability distribution of correlation, measuring the weight of a given Fourier wave mode $k$ in the power spectrum [9]. Hence, the CE is responsible for measuring the amount of information that is encrypted in
TABLE II. The CE for the pseudoscalar pion family, respectively in each column for the quadratic and deformed dilatonic field backgrounds.

\begin{tabular}{lcc}
\hline \hline$n$ & pion CE $\left(\phi_{1}(\mathrm{z})\right)$ & pion CE $\left(\phi_{2}(\mathrm{z})\right)$ \\
\hline 1 & 97.5 & 77.9 \\
2 & 702.1 & 506.3 \\
3 & $5.613 \times 10^{3}$ & $3.129 \times 10^{3}$ \\
4 & $2.587 \times 10^{4}$ & $1.548 \times 10^{4}$ \\
5 & $1.490 \times 10^{5}$ & $1.275 \times 10^{5}$ \\
\hline \hline
\end{tabular}

the spatial portrait of the energy density. The CE reads $[1,2,20,21]$

$$
S[\epsilon]=-\int_{\mathbb{R}} \widetilde{\epsilon}_{\bullet}(k) \log \widetilde{\epsilon} \cdot(k) d k,
$$

for $\widetilde{\epsilon}_{\bullet}(k)=\epsilon_{\bullet}(k) / \epsilon_{\cdot \max }(k)$. Following this protocol, the CE can be computed for the pseudoscalar pion meson family, first as a function of the excitation number $n$. Table II shows the CE for both dilatonic field backgrounds (15)-(16). Table II shows the $\mathrm{CE}$ of the pseudoscalar pion family, identifying the $\pi_{n}$ eigenfunctions in Eq. (12), as $\pi_{1}=\left\{\pi_{ \pm}, \pi_{0}\right\}, \pi_{2}=\pi(1300), \pi_{3}=\pi(1800), \pi_{4}=\pi(2070)$, $\pi_{5}=\pi(2360)$. Then, the CE of each $\pi_{n}$ meson mode in the pion meson family is represented by the points in Fig. 2, strictly for the $\pi$ meson PDG values. In addition, introducing linear regression yields the configurational entropic Regge trajectory, for the pion family, represented by the dashed line in Fig. 2. The explicit expression of the configurational entropic Regge trajectory reads

$$
\log \left(\mathrm{CE}_{\pi}(n)\right)=1.8256 n+2.8927
$$

within $\sim 1 \%$ standard deviation. Similarly, the CE can be also computed, now taking into account the $\phi_{2}$ dilaton (16), illustrated in the third column of Table II. Figure 3 depicts

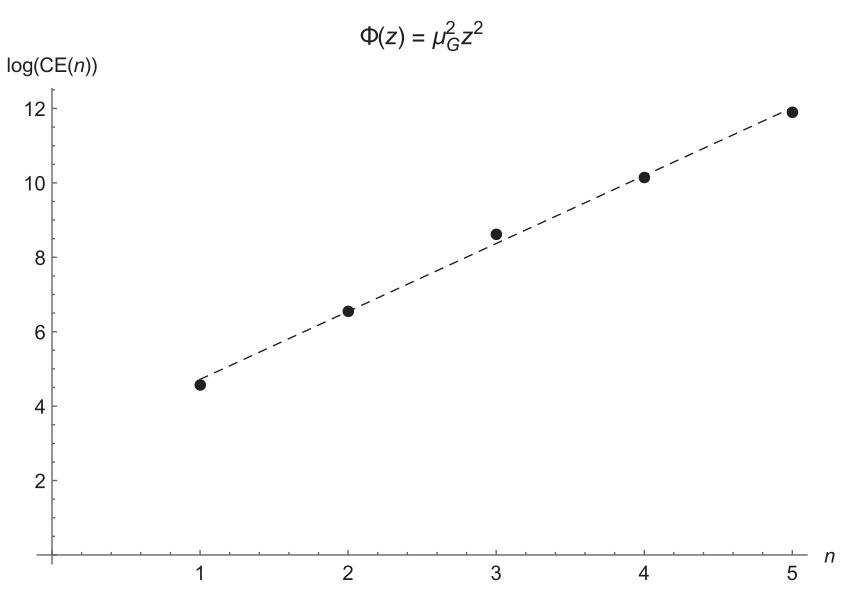

FIG. 2. CE of the pion family as a function of the quantum number $n$, in the quadratic dilaton AdS/QCD soft-wall model. 


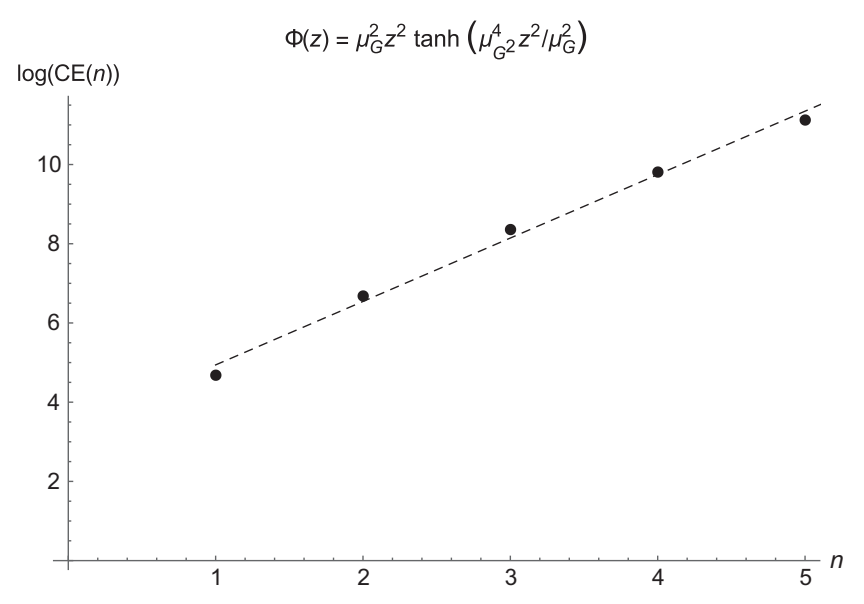

FIG. 3. CE of the pion family as a function of the quantum number $n$, in the deformed dilaton AdS/QCD soft-wall model.

the computed results based on the PDG values for the $\pi_{n}$ pion modes $(n \leq 5)$ in Table II, illustrating the configurational entropic Regge trajectory in the deformed dilaton AdS/QCD soft-wall model. Linear regression yields the following relation between the configurational entropy of the pion family and the excitation number $n$ :

$$
\log \left(\mathrm{CE}_{\pi}(n)\right)=1.6015 n+3.3398,
$$

within $\sim 0.2 \%$ standard deviation.

A scaling law can be derived from Eqs. (22)-(23). This is reflected in the configurational entropic Regge trajectory, which in this case associates the CE with the excitation number $n$, for the $\pi$ meson family. Motivated by the standard Regge trajectory in Fig. 1, that relates the $\pi$ meson squared mass spectra to the excitation number $n$, one can apply this method in reverse to predict the mass spectra of the other members of the $\pi$ meson family, that have not been experimentally observed. Indeed, computing the CE for the meson family with respect to the $\pi_{n}$ meson family mass, once the respective excitation $n$ is fixed, yields data that can be interpolated to determine the masses of the other members of the $\pi$ meson family, that have not been experimentally detected.

First, the quadratic dilaton (15) is used to infer the mass spectra of the $\pi_{n}$ meson family, in the top panel of Fig. 4. The deformed dilatonic field (16) is a refined model that implements the mass spectra of the $\pi$ meson family, in great agreement with PDG values, having the quadratic dilaton (15) as the UV limit. The configurational entropic Regge trajectory, with respect to the $\pi$ meson family mass, is then shown in the bottom panel of Fig. 4. Respectively for the plots in Fig. 4, the configurational entropic Regge trajectories have the following explicit expressions:

$$
\log \left(\mathrm{CE}_{\pi}(m)\right)=1.5337 \times 10^{-6} m^{2}-0.0005 m+4.6301,
$$

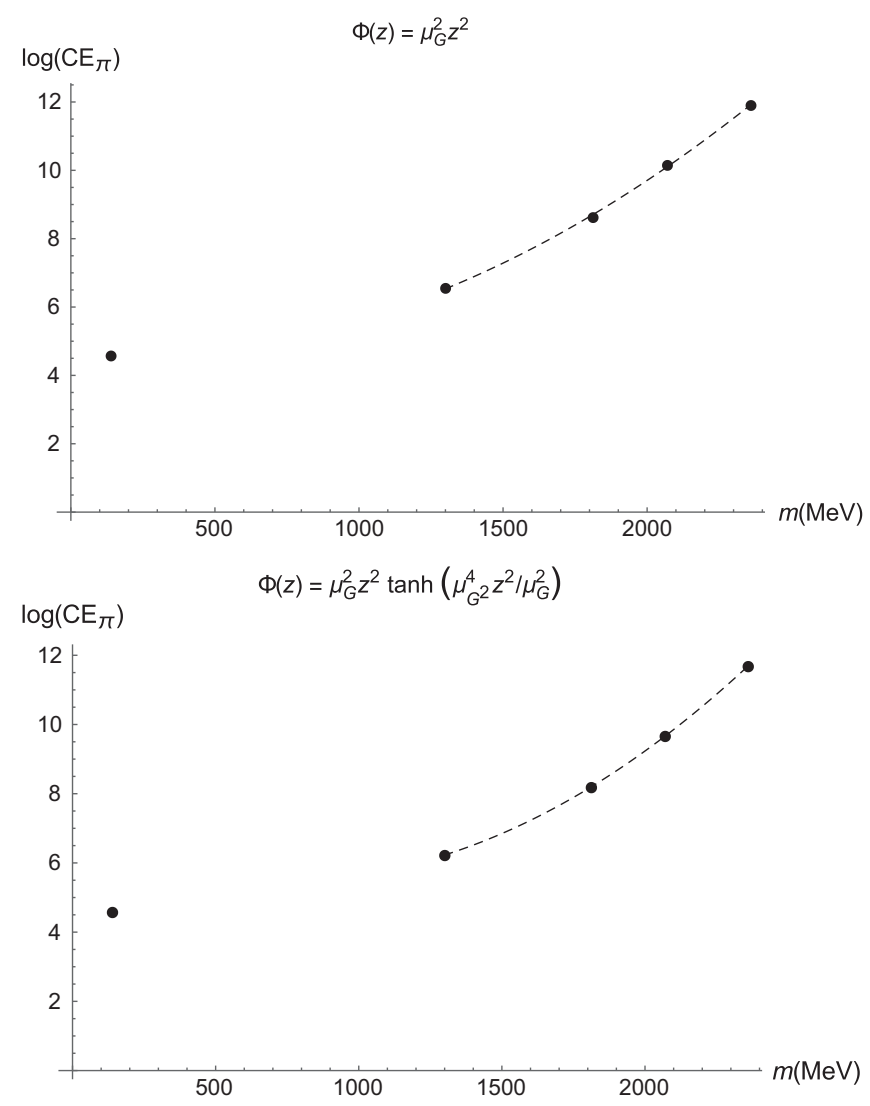

FIG. 4. Configurational entropic Regge trajectory of the $\pi$ meson family with respect to the mass spectra, using the quadratic dilaton (top panel) and deformed dilaton (bottom panel).

$$
\log \left(\mathrm{CE}_{\pi}(m)\right)=2.3869 \times 10^{-6} m^{2}-0.0036 m+5.8556,
$$

within $\sim 0.2 \%$ standard deviation. We introduce a procedure that is alternative to solving the system (11)-(12), to calculate the mass spectra of the $\pi$ meson family. Using the linear regression (22)-(23) and the quadratic interpolation (24)-(25), one can calculate the CE of the $\pi$ meson family, for the $\pi_{n}$ excitations with $n>5$, with good accuracy for the members $\pi_{n}$ of the pion meson family that are subsequent to the $\pi_{5}$ element.

We denote by $m_{\pi, n}$ the associated mass spectrum of the $n$th pion, in the pseudoscalar $\pi$ meson family, For the quadratic dilaton, the mass spectra of the $\pi_{6}, \pi_{7}$ and $\pi_{8}$ members of the $\pi$ meson family can be then inferred. In fact, for $n=6$, Eq. (22) yields $\log \left(\mathrm{CE}_{\pi}\right)=13.846$. Substituting this value into the configurational entropic Regge trajectory (24), one obtains the mass $m_{\pi, 6}=2630 \mathrm{MeV}$, for the $\pi_{6}$ mesonic state, yielding the reliable range $2612 \mathrm{MeV} \lesssim m_{\pi, 6} \lesssim 2648 \mathrm{MeV}$, for the $\pi_{6}$ pseudoscalar meson state. Analogously, the $\pi_{7}$ meson has a derived mass $m_{\pi, 7}=2861 \mathrm{MeV}$. The standard deviations of Eqs. (22) and (24) then yield the reliable mass range $2839 \lesssim m_{\pi, 7} \lesssim 2883 \mathrm{MeV}$. Once more, the $\pi_{8}$ 
pseudoscalar state has mass $m_{\pi, 8}=3074 \mathrm{MeV}$, and experimental standard deviations yield the trustworthy range $3049 \lesssim m_{\pi, 7} \lesssim 3099 \mathrm{MeV}$. On the other hand, for the deformed dilaton, considering $n=6$ in Eq. (22) implies that $\log \left(\mathrm{CE}_{\pi}\right)=12.948$. Substituting this into the configurational entropic Regge trajectory (24) yields the mass $m_{\pi, 6}=2631 \mathrm{MeV}$, within the experimentally measured range $2612 \mathrm{MeV} \lesssim m_{\pi, 6} \lesssim 2649 \mathrm{MeV}$, for the $\pi_{6}$ pseudoscalar meson. Analogously, one can derive for the $\pi_{7}$ mesonic state the mass $m_{\pi, 7}=2801 \mathrm{MeV}$, within the reliable range $2779 \lesssim m_{\pi, 7} \lesssim 2823 \mathrm{MeV}$, considering standard deviations in Eqs. (22) and (24). In addition, the $\pi_{8}$ meson presents a mass equal to $m_{\pi, 8}=2959 \mathrm{MeV}$, establishing the phenomenologically reliable range $2934 \lesssim m_{\pi, 8} \lesssim 2984 \mathrm{MeV}$ for its mass.

\section{CONCLUDING REMARKS AND PERSPECTIVES}

The main feature that distinguishes the $\pi$ meson family description in AdS/QCD from other light-flavor meson families is the EOMs that govern the mesonic state modes and their mass spectra for each meson family. In fact, delving into the pions one can realize that they are the only family along with the $\phi$ mesons, among the $a_{1}, f_{0}$, and $\rho$ mesons in AdS/QCD, whose EOM (11) has a potential that is a function of another meson, the $\phi$ meson. In this case, there is a coupling between the EOMs of the $\pi$ and the $\phi$ mesons. This reflects a nonlinear configurational entropic Regge trajectory for the pion family, with respect to the $\pi$ meson family mass, as shown in the plots in Fig. 4, as well as the analytical expressions of the CE for these plots, in Eqs. (24)-(25). In addition, the configurational entropic Regge trajectory, for the pion family, is linear with respect to the excitation number $n$, as shown in the plots in Fig. 4, and given by the explicit analytical expressions (22)-(23). This is a common feature of the $f_{0}, a_{1}$ and $\rho$ meson families studied in Ref. [17]. However, for these meson families the configurational entropic Regge trajectories, as functions of the respective meson family mass spectra, are also linear, contrary to the case analyzed here and depicted in the plots in Fig. 4. It is then worth emphasizing that the reason for the nonlinearity of the configurational entropic Regge trajectory for the pion family, with respect to the $\pi$ meson mass spectra, is the coupling between the EOMs (11)-(12), with the respective coupled potentials (13)-(14).

Another piece of information provided by the configurational entropic Regge trajectories is the values of the masses of the next generation of the $\pi$ states. Using the CE value of the $n$th excitation, Eqs. (22)-(23), one can employ Eqs. (22) and (24) to infer the mass spectra of the $\pi_{6}, \pi_{7}$ and $\pi_{8}$ mesons, as discussed throughout Sec. III. In the case of the quadratic dilaton the results found were $m_{\pi, 6}=2630 \pm 18 \mathrm{MeV}$, $m_{\pi, 7}=2861 \pm 22 \mathrm{MeV}$ and $m_{\pi, 8}=3074 \pm 25 \mathrm{MeV}$. On the other hand, for the deformed dilaton the masses found were $m_{\pi, 6}=2631 \pm 18 \mathrm{MeV}, m_{\pi, 7}=2801 \pm 22 \mathrm{MeV}$ and $m_{\pi, 8}=2959 \pm 25 \mathrm{MeV}$. It is possible to improve these values of the masses with the eventual detection of the pion excitation states, that shall contribute with more experimental points in Fig. 1.

\section{ACKNOWLEDGMENTS}

L. F. is supported by the National Council for Scientific and Technological Development-CNPq (Brazil) under Grant No. 153337/2018-4. R.d.R. is grateful to FAPESP (Grant No. 2017/18897-8) and to CNPq (Grant No. 303293/2015-2), for partial financial support.
[1] M. Gleiser and N. Stamatopoulos, Phys. Lett. B 713, 304 (2012).

[2] M. Gleiser and N. Stamatopoulos, Phys. Rev. D 86, 045004 (2012).

[3] A. E. Bernardini and R. da Rocha, Phys. Lett. B 762, 107 (2016).

[4] A. E. Bernardini, N. R. F. Braga, and R. da Rocha, Phys. Lett. B 765, 81 (2017).

[5] A. Goncalves and R. da Rocha, Phys. Lett. B 774, 98 (2017).

[6] N. R. F. Braga and R. da Rocha, Phys. Lett. B 776, 78 (2018).

[7] C. W. Ma and Y. G. Ma, Prog. Part. Nucl. Phys. 99, 120 (2018).

[8] N. Barbosa-Cendejas, R. Cartas-Fuentevilla, A. HerreraAguilar, R. R. Mora-Luna, and R. da Rocha, Phys. Lett. B 782, 607 (2018).
[9] N. R. F. Braga, L. F. Ferreira, and R. da Rocha, Phys. Lett. B 787, 16 (2018).

[10] G. Karapetyan, Europhys. Lett. 117, 18001 (2017).

[11] G. Karapetyan, Europhys. Lett. 118, 38001 (2017).

[12] G. Karapetyan, Phys. Lett. B 781, 201 (2018).

[13] G. Karapetyan, Phys. Lett. B 786, 418 (2018).

[14] G. Karapetyan, arXiv:1901.05349 [Europhys. Lett. (to be published)].

[15] A. Alves, A. G. Dias, and R. da Silva, Physica 420, 1 (2015).

[16] A. Alves, A. G. Dias, and R. da Silva, Braz. J. Phys. 47, 426 (2017).

[17] A. E. Bernardini and R. da Rocha, Phys. Rev. D 98, 126011 (2018).

[18] M. Gleiser and N. Graham, Phys. Rev. D 89, 083502 (2014).

[19] M. Gleiser and D. Sowinski, Phys. Lett. B 747, 125 (2015).

[20] M. Gleiser and D. Sowinski, Phys. Lett. B 727, 272 (2013). 
[21] M. Gleiser and N. Jiang, Phys. Rev. D 92, 044046 (2015).

[22] R. A. C. Correa and R. da Rocha, Eur. Phys. J. C 75, 522 (2015).

[23] R. A. C. Correa, D. M. Dantas, C. A. S. Almeida, and R. da Rocha, Phys. Lett. B 755, 358 (2016).

[24] D. Sowinski and M. Gleiser, J. Stat. Phys. 167, 1221 (2017).

[25] R. Casadio and R. da Rocha, Phys. Lett. B 763, 434 (2016).

[26] N. R. F. Braga and R. da Rocha, Phys. Lett. B 767, 386 (2017).

[27] M. Gleiser, M. Stephens, and D. Sowinski, Phys. Rev. D 97, 096007 (2018).

[28] C. O. Lee, Phys. Lett. B 790, 197 (2019).

[29] D. Bazeia, D. C. Moreira, and E. I. B. Rodrigues, J. Magn. Magn. Mater. 475, 734 (2019).

[30] E. Witten, arXiv:1805.11965.

[31] A. Karch, E. Katz, D. T. Son, and M. A. Stephanov, Phys. Rev. D 74, 015005 (2006).

[32] P. Zhang, J. High Energy Phys. 05 (2010) 039.

[33] P. Colangelo, F. Giannuzzi, S. Nicotri, and V. Tangorra, Eur. Phys. J. C 72, 2096 (2012).

[34] P. Colangelo and F. Loparco, Phys. Lett. B 788, 500 (2019).

[35] D. Li and M. Huang, J. High Energy Phys. 11 (2013) 088.

[36] S. J. Brodsky, G. F. de Teramond, H. G. Dosch, and J. Erlich, Phys. Rep. 584, 1 (2015).

[37] L. Da Rold and A. Pomarol, Nucl. Phys. B721, 79 (2005).

[38] T. Gherghetta, J. I. Kapusta, and T. M. Kelley, Phys. Rev. D 79, 076003 (2009).

[39] J. M. Maldacena, Int. J. Theor. Phys. 38, 1113 (1999); Adv. Theor. Math. Phys. 2, 231 (1998).

[40] J. Polchinski and M. J. Strassler, Phys. Rev. Lett. 88, 031601 (2002).

[41] H. Boschi-Filho and N. R. F. Braga, Eur. Phys. J. C 32, 529 (2004).
[42] H. Boschi-Filho and N. R. F. Braga, J. High Energy Phys. 05 (2003) 009.

[43] J. Erlich, E. Katz, D. T. Son, and M. A. Stephanov, Phys. Rev. Lett. 95, 261602 (2005).

[44] H. R. Grigoryan and A. V. Radyushkin, Phys. Rev. D 76, 115007 (2007).

[45] S. J. Brodsky and G. F. de Teramond, Phys. Rev. D 77, 056007 (2008).

[46] Y.-Q. Sui, Y.-L. Wu, Z.-F. Xie, and Y.-B. Yang, Phys. Rev. D 81, 014024 (2010).

[47] T. M. Kelley, S. P. Bartz, and J. I. Kapusta, Phys. Rev. D 83, 016002 (2011).

[48] A. Ballon-Bayona, G. Krein, and C. Miller, Phys. Rev. D 91, 065024 (2015).

[49] M. Lv, D. Li, and S. He, arXiv:1811.03828.

[50] M. Tanabashi et al. (Particle Data Group), Phys. Rev. D 98, 030001 (2018).

[51] P. Colangelo, F. De Fazio, F. Giannuzzi, F. Jugeau, and S. Nicotri, Phys. Rev. D 78, 055009 (2008).

[52] S. S. Afonin, Phys. Lett. B 719, 399 (2013).

[53] N. R. F. Braga, M. A. M. Contreras, and S. Diles, Phys. Lett. B 763, 203 (2016).

[54] N. R. F. Braga and L. F. Ferreira, Phys. Lett. B 783, 186 (2018).

[55] C. A. B. Bayona, H. Boschi-Filho, and N. R. F. Braga, J. High Energy Phys. 03 (2008) 064.

[56] W. de Paula, T. Frederico, H. Forkel, and M. Beyer, Phys. Rev. D 79, 075019 (2009).

[57] W. de Paula and T. Frederico, Phys. Lett. B 693, 287 (2010).

[58] R. Rougemont, R. Critelli, J. Noronha-Hostler, J. Noronha, and C. Ratti, Phys. Rev. D 96, 014032 (2017). 